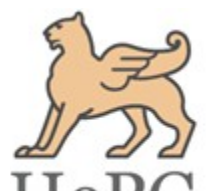
HePG

\title{
An update on sequenced chloroplast genomes of Bryophytes
}

\author{
Asheesh Shanker \\ Department of Bioscience and Biotechnology, Banasthali University, Rajasthan, India
}

\section{Article history}

Received: 19 August 2015

Accepted: 10 September 2015

Published online: 1 October 2015

(C) Shanker (2015)

Editors

Afroz Alam

Dipjyoti Chakraborty

Publisher

Horizon e-Publishing Group

Corresponding Author

Asheesh Shanker

凶ashomics@gmail.com

\begin{abstract}
The sequencing of complete chloroplast genome of Marchantia polymorpha paved the way to know the structure and organization of chloroplast genomes of other plants. Since then 747 chloroplast genomes have been sequenced. However, in comparison to the tracheophytes, a small number of complete chloroplast genome sequences of bryophytes are available (only 4 liverworts, 6 mosses and 2 hornwort). This review represents an update on sequenced chloroplast genomes of bryophytes.
\end{abstract}

Keywords

Bryophytes; hornworts; liverworts; mosses; chloroplast genome

Shanker, A. 2015. An update on sequenced chloroplast genomes of Bryophytes. Plant Science Today 2(4): 172-174. http://dx.doi.org/10.14719/pst.2015.2.4.14 $\underline{3}$

\section{Introduction}

Bryophytes are the earliest and the simplest land plants. The extant lineages of bryophytes are classified into hornworts, liverworts, and mosses. Phylogenetic analysis based on chloroplast and mitochondrial genome sequences confirmed paraphyletic origin of bryophytes (Shanker, 2013a; 2013b; 2013c). Green plants contain chloroplasts as an intracellular organelles which have their own autonomously replicating genome. It encode a number of components for the process of photosynthesis (Sugiura, 1989). Among all land plants the first chloroplast genome sequenced was that of a bryophyte, Marchantia polymorpha (Ohyama et al,. 1986; Ohyama et al., 1988). However, the chloroplast genome sequencing of bryophytes was outpaced by other plants. Consequently only 12 chloroplast genomes of bryophytes are available in public repositories among 747 total chloroplast genome sequences of green plants (Kapil et al., 2014). These chloroplast genome sequences belong to algae, bryophytes, pteridophytes, gymnosperms, and angiosperms.
Previously a review on sequenced chloroplast genomes of bryophytes including 3 liverworts: Aneura mirabilis, Marchantia polymorpha, Ptilidium pulcherrimum; 2 Mosses: Physcomitrella patens, Syntrichia ruralis, and 1 Hornwort: Anthoceros formosae was published (Shanker, 2012). Since then only 6 new chloroplast genome sequences (3 published and 3 unpublished) have been added in public repository at National Center for Biotechnology Information (NCBI). A list of available chloroplast genomes of bryophytes is given in Table 1. The present review is an update on newly sequenced chloroplast genome of bryophytes (1 each of liverwort, moss, and hornwort).

\section{Pellia endiviifolia (Dicks.) Dumort.} (Marchantiophyta)

The chloroplast genome of Pellia endiviifolia consist of 120546 base pairs (bp) with 123 genes, detected based on orthologous relationship from other chloroplast genomes. A strong conservation in the gene content, gene order and overall arrangement was observed in comparison to the chloroplast genome of 
Table 1. Sequenced chloroplast genomes of bryophytes available at NCBI.

\begin{tabular}{|c|c|c|c|c|}
\hline S. No. & Organism Name & *Accession No. & $\begin{array}{l}\text { Genome Size } \\
\text { (bp) }\end{array}$ & Reference \\
\hline & $\begin{array}{l}\text { Marchantiophyta } \\
\text { (Liverworts) }\end{array}$ & & & \\
\hline 1. & Aneura mirabilis & NC_010359 & 108007 & Wickett et al. 2008 \\
\hline 2. & Marchantia polymorpha & NC_001319 & 121024 & Ohyama et al. 1986 \\
\hline 3. & Pellia endiviifolia & NC_019628 & 120546 & Grosche et al. 2012 \\
\hline \multirow[t]{2}{*}{4.} & Ptilidium pulcherrimum & NC_015402 & 119007 & Forrest et al. 2011 \\
\hline & Bryophyta (Mosses) & & & \\
\hline 5. & Nyholmiella obtusifolia & NC_026979 & 122895 & NCBI \\
\hline 6. & Orthotrichum rogeri & NC_026212 & 123363 & NCBI \\
\hline 7. & Physcomitrella patens & NC_005087 & 122890 & Sugiura et al. 2003 \\
\hline 8. & Sanionia uncinata & NC_025668 & 124374 & NCBI \\
\hline 9. & Syntrichia ruralis & NC_012052 & 122630 & Oliver et al. 2010 \\
\hline \multirow[t]{2}{*}{10.} & Tetraphis pellucida & NC_024291 & 127489 & Bell et al. 2014 \\
\hline & $\begin{array}{l}\text { Anthocerotophyta } \\
\text { (Hornworts) }\end{array}$ & & & \\
\hline 11. & Anthoceros formosae & NC_004543 & 161162 & Kugita et al. 2003 \\
\hline 12. & Nothocerosaenigmaticus & NC_020259 & 153208 & Villarreal et al. 2013 \\
\hline
\end{tabular}

*Chloroplast genome sequence at NCBI will be accessed using this number.

Marchantia polymorpha. Moreover, the sequences on the borders of inverted repeats, small single copy, and large single copy were also conserved. The overall A/T content of $64.11 \%$ was reported. Pellia endiviifolia possess more compact inverted repeat regions, a $301 \mathrm{bp}$ long non-coding region at the border between inverted repeat $\mathrm{b}$ and 3' rps12, and a $550 \mathrm{bp}$ deletion in the coding region of ycf2. Apart from these differences, 21 introns were also identified in the chloroplast genome of Pellia endiviifolia (Grosche et al., 2012).

\section{Tetraphis pellucida Hedw. (Bryophyta)}

The chloroplast genome sequence of four-toothed moss, Tetraphis pellucida, comprises of $127489 \mathrm{bp}$ and its genome structure was found similar with other available organellar genomes. It possess inverted repeat regions of 9564 bp separated by a small single copy region (18927 bp) and a large single copy region
(89434 bp). The GC content was $29.4 \%$ which is similar to chloroplast genomes of bryophytes, however, $34-40 \%$ lesser than found in seed plants.

Common with Syntrichia ruralis, the chloroplast genome of Tetraphis pellucida lacks the petN and rpoA genes and the inversion of around $71 \mathrm{~kb}$ in the large single copy region. Moreover, the gene content in the inverted repeats of both these species were found identical. Due to an increased total length of intragenic spacer regions in the large single copy region of the Tetraphis pellucida chloroplast genome, it is $\sim 5 \mathrm{~kb}$ longer than those of Syntrichia ruralis and Physcomitrella patens (Bell et al., 2014).

Nothoceros aenigmaticus (R.M. Schust.) J.C. Villarreal \& K.D. McFarland (Anthocerotophyta)

Earlier only a single chloroplast genome sequence of the hornwort Anthoceros formosae was available (Kugita et al., 2003). In comparison to other 
bryophytes it possess an expanded inverted repeat and a type I intron in the $23 \mathrm{~S}$ ribosomal RNA gene. Recently shotgun sequencing of genomic DNA was used to sequence the chloroplast genome of another hornwort, Nothoceros aenigmaticus, and it was found colinear with chloroplast genome sequences of other bryophytes (Villarreal et al., 2013). The chloroplast DNA of this hornwort contains 153208 bp out of which 11732 bp belongs to each inverted repeat. It contains a total of 124 genes (88 codes for proteins, 32 transfer RNAs and 4 ribosomal RNAs). The bases were found in the following proportion: $32.7 \%(\mathrm{~A})$, $17.1 \%(\mathrm{G}), 17.9 \%(\mathrm{C})$, and $32.3 \%(\mathrm{~T})$ with a GC content of $\sim 35 \%$. The genes trnI CAU and trnV GAC are the terminal genes of the inverted repeat. The maturase $\mathrm{K}$ gene (matK), annotated as a pseudogene in the chloroplast genome of Anthoceros formosae, was found as seemingly functional. Three structural differences were observed when the chloroplast genome sequence of Nothoceros aenigmaticus was compared with that of Anthoceros. Many genes found within the inverted repeat in Anthoceros were located in the large single copy region in Nothoceros. Moreover, the rpl2 gene was found as a pseudogene and there is a lack of an intron in the rrn23 gene (Villarreal et al. 2013).

As a concluding remark, despite the importance of bryophytes in the early evolution of land plants their complete nuclear, chloroplast and mitochondrial (Kumar et al., 2014) genomes are poorly represented in public databases. Therefore, efforts are required for the genome sequencing of these early land plants which will in turn help to further solve the mystery of plants adaptation on land.

\section{Acknowledgements}

I am thankful to Dr. Afroz Alam, Associate Professor, Banasthali University, Rajasthan for useful comments.

\section{References}

Bell, N. E., J. L. Boore, B. D. Mishler, and J. Hyvonen. 2014. Organellar genomes of the four-toothed moss, Tetraphis pellucida. BMC Genomics 15: 383.

Forrest, L. L., N. J. Wickett, C. J. Cox, and B. Goffinet. 2011. Deep sequencing of Ptilidium (Ptilidiaceae) suggests evolutionary stasis in liverwort plastid genome structure. Plant Ecol Evol 144: 29-43.

Grosche, C., H. T. Funk, U. G. Maier, and S. Zauner. 2012. The chloroplast genome of Pellia endiviifolia: gene content, RNA-editing pattern, and the origin of chloroplast editing. Genome Biol Evol 4: 1349-1357.

Kapil, A., P. K. Rai, and A. Shanker. 2014. ChloroSSRdb: a repository of perfect and imperfect chloroplastic simple sequence repeats (cpSSRs) of green plants. Database (Oxford) 2014 doi: 10.1093/database/bau107.

Kugita, M., A. Kaneko, Y. Yamamoto, Y. Takeya, T. Matsumoto, and K. Yoshinaga. 2003. The complete nucleotide sequence of the hornwort (Anthoceros formosae) chloroplast genome: insight into the earliest land plants. Nucleic Acids Res 31: 716-721.

Kumar, M., A. Kapil, and A. Shanker. 2014. MitoSatPlant: mitochondrial microsatellites database of viridiplantae. Mitochondrion 19: 334-337.
Ohyama, K., H. Fukuzawa, T. Kohchi, H. Shirai, T. Sano, S. Sano, K. Umesono, Y. Shiki, M. Takeuchi, Z. Chang, S. Aota, H. Inokuchi, and H. Ozeki. 1986. Chloroplast gene organization deduced from complete sequence of liverwort Marchantia polymorpha chloroplast DNA. Nature 322: 572- 574.

Ohyama, K., H. Fukuzawa, T. Kohchi, T. Sano, S. Sano, H. Shirai, K. Umesono, Y. Shiki, M. Takeuchi, Z. Chang, H. Inokuchi, and H. Ozeki. 1988. Structure and organization of Marchantia polymorpha chloroplast genome. I. Cloning and gene identification. $\mathrm{J} \mathrm{Mol}$ Biol 203: 281-298.

Oliver, M.J., A. G. Murdock, B. D. Mishler, J. V. Kuehl, J. L. Boore, D. F. Mandoli, K. D. Everett, P. G. Wolf, A. M. Duffy, and K. G. Karol. 2010. Chloroplast genome sequence of the moss Tortula ruralis: gene content, polymorphism, and structural arrangement relative to other green plant chloroplast genomes. $B M C$ Genomics 11: 143.

Shanker, A. 2012. Chloroplast Genomes of Bryophytes: A Review. Arch Bryol 143: 1-5.

Shanker, A. 2013a. Paraphyly of bryophytes inferred using chloroplast sequences. Arch Bryol 163: 1-5.

Shanker, A. 2013b. Inference of bryophytes paraphyly using mitochondrial genomes. Arch Bryol 165: 1-5.

Shanker, A. 2013c. Combined data from chloroplast and mitochondrial genome sequences showed paraphyly of bryophytes. Arch Bryol 171: 1-9.

Sugiura, C. Y., S. Kobayashi, Aoki, C. Sugita, and M. Sugita. 2003. Complete chloroplast DNA sequence of the moss Physcomitrella patens: evidence for the loss and relocation of rpoA from the chloroplast to the nucleus. Nucleic Acids Res 31: 5324-5331.

Sugiura, M. 1989. The chloroplast chromosomes in land plants. Annu Rev Cell Biol 5: 51-70.

Villarreal, J. C., L. L. Forrest, N. Wickett, and B. Goffinet. 2013: The plastid genome of the hornwort Nothoceros aenigmaticus (Dendrocerotaceae): phylogenetic signal in inverted repeat expansion, pseudogenization, and intron gain. Am J Bot 100: 467-477.

Wickett, N.J., Y. Zhang, S. K. Hansen, J. M. Roper, J. V. Kuehl, S. A. Plock, P. G. Wolf, C. W. DePamphilis, J. L. Boore, and Goffinet B. 2008. Functional gene losses occur with minimal size reduction in the plastid genome of the parasitic liverwort Aneura mirabilis. Mol Biol Evol 25: 393-401.

$$
\text { 사 }
$$

